\title{
A Fast and Retargetable Framework for Logic-IP-Internal Electromigration Assessment Comprehending Advanced Waveform Effects
}

\author{
Palkesh Jain, Member, IEEE, Jordi Cortadella, Fellow, IEEE, and Sachin S. Sapatnekar, Fellow, IEEE
}

\begin{abstract}
A new methodology for system-on-chip-level logic-IP-internal electromigration verification is presented in this paper, which significantly improves accuracy by comprehending the impact of the parasitic $R C$ loading and voltage-dependent pin capacitance in the library model. It additionally provides an on-the-fly retargeting capability for reliability constraints by allowing arbitrary specifications of lifetimes, temperatures, voltages, and failure rates, as well as interoperability of the IPs across foundries. The characterization part of the methodology is expedited through the intelligent IP-response modeling. The ultimate benefit of the proposed approach is demonstrated on a 28-nm design by providing an on-the-fly specification of retargeted reliability constraints. The results show a high correlation with SPICE and were obtained with an order of magnitude reduction in the verification runtime.
\end{abstract}

Index Terms-Electromigration (EM), pin capacitance, reliability, retargeting, signal probability.

\section{INTRODUCTION}

$\mathbf{E}$ LECTROMIGRATION (EM) is a major product aging mechanism revolving around the containment of the average and rms current densities in interconnects. This, in turn, requires a cell-external analysis for signals and power nets connecting to the cells and a cell-internal analysis for wires within a logic-IP (standard cells) or mixed signal IP block. Recently, a great deal of innovation and improvement has been seen on the verification and design strategies for cell-external signal and power grid EM [1]-[4]. However, there has not been an adequate focus on the robust design and reuse of the standard cells. Ensuring EM reliability for standard cells and IPs in a design implies that the exact context at which the IP is used must be bounded to guarantee its robustness in the design. This context could be stated in terms of design limits (loads, slews, frequencies, and supply voltage), or reliability (temperature, lifetime, or a failure rate specification tied to current density limits). Without rigorous assessments, a set of IPs designed for a particular reliability condition [e.g., $1.2 \mathrm{~V}, 105{ }^{\circ} \mathrm{C}, 100 \mathrm{k}$ power-on hours $(\mathrm{POH}), 0.1 \%$

Manuscript received June 19, 2015; revised October 12, 2015; accepted November 27, 2015. This work was supported in part under NSF award CCF1162267.

P. Jain was with Texas Instruments India Pvt, Ltd., Bangalore 560093, India. $\mathrm{He}$ is now with Qualcomm Technologies Inc., Bangalore 560066, India (email: palkesh@qti.qualcomm.com).

J. Cortadella is with the Computer Science Department, Universitat Politècnica de Catalunya, Barcelona 08034, Spain (e-mail: jordi.cortadella@upc.edu).

S. S. Sapatnekar is with the University of Minnesota, Minneapolis, MN 55455 USA (e-mail: sachin@umn.edu).

Color versions of one or more of the figures in this paper are available online at http://ieeexplore.iee.org.

Digital Object Identifier 10.1109/TVLSI.2015.2505504

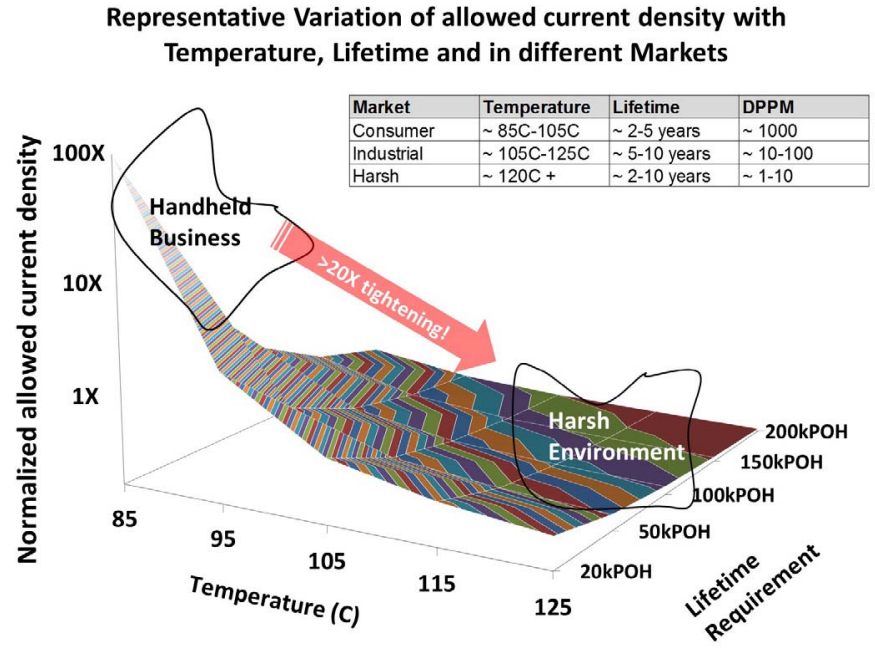

Fig. 1. Typical current density limits as a function of temperature and lifetime, showing $>20 \times$ differences between various environments.

cumulative failure, and $10{ }^{\circ} \mathrm{C}$ Joule heating $(\mathrm{JH})$ limit] cannot be guaranteed to be EM-safe at another condition (e.g., $1.0 \mathrm{~V}, 115{ }^{\circ} \mathrm{C}, 200 \mathrm{k} \mathrm{POH}, 0.01 \%$ cumulative failure, and $15^{\circ} \mathrm{C} \mathrm{JH}$ limit).

Nevertheless, the tradeoffs on these constraints are increasingly in demand in industry due to accelerated inroads of semiconductor houses into newer businesses with different reliability demands [5]. For example, industrial designs demand more stringent operating conditions than traditional computing applications [6], [7]. From an EM standpoint, meeting these specifications is challenging, as shown in Fig. 1, which highlights the representative current density per $\mu \mathrm{m}^{2}$ across various temperature and lifetime specifications. As can be seen, among the various environments, the current carrying capability becomes over $20 \times$ more stringent. Not only among different application markets, even for the same system-onchip (SoC) itself, different complex IPs (e.g., CPU core or a DSP) can have different reliability requirements based on their ON times and temperature specifications. The challenges increase when such reliability requirements could only be made available on the fly: that is, either during the final SoC verification or even after the SoC tape-out; in which cases, the original reliability targets for the IP, characterized for one application domain, may not match with the reliability requirements in a different domain.

One way to meet such diverse specifications is to approach the design in a bottom-up manner with a fresh logic-IP portfolio that meets targeted domain-specific reliability specifications. However, this is very expensive, and economic 


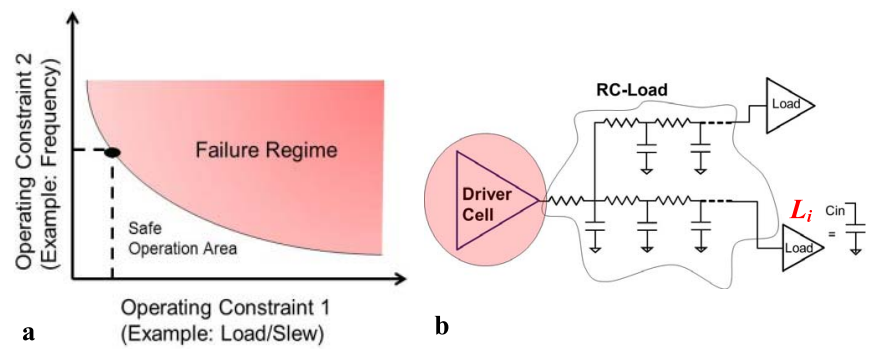

Fig. 2. (a) Traditional approach for EM verification using the safe operating region concept. (b) Schematic highlighting the EM-critical cell, driving an $R C$ load network (vis-à-vis safe frequency obtained for pure $C$ load)

and design effort considerations often dictate that the product integration over all application domains be based on the same IP portfolio. This implies that the logic-IPs require a disciplined utilization procedure, making it important to assess their exact usage boundaries at arbitrary conditions.

A starting point toward this is to ensure that the cell is EM-safe at a specific load and frequency by selecting wire widths, so that EM constraints are met. However, this only implies that a lower load and lower frequency can be considered EM-safe. The cell may (or may not) be EM-safe at a lower load and higher frequency, or a higher load and lower frequency, or a higher load and higher frequency, and this can only be uncovered through costly detailed analysis.

As an improvement, some industrial implementation tools [8], [9] use a precharacterized table that models the tradeoffs in various design/reliability parameters. Fig. 2(a) shows a representation of one such table, where the $x$-axis represents an operating constraint of the cell (load here, but this could be slew, supply voltage, or any reliability constraint) and the $y$-axis represents the alternative constraint (frequency here), at a baseline reliability condition.

The intuition behind such a table (frequency versus load; $f-L)$ is simple: the current flow in the IP increases with the operating load, and hence, the frequency should be lowered to meet the reliability specification. This model can be used at the chip level to determine the safe frequency ( $\left.f_{\text {safe }}\right)$ of an instance for any design/reliability parameter, and then make corresponding design fixes. Needless to say, most of the EM-critical cells are the ones that operate at higher loads, frequencies, or slews.

However, such a specification is also simplistic and with advancing technology and convoluted circuit effects, and this model is inadequate in accurately predicting EM safety for several reasons. First, the frequency in Fig. 2(a) refers to the output switching frequency: for multi-input cells, the failure rate depends on the switching frequency at each input. This corresponds to a multidimensional space that is computationally expensive to characterize. Second, EM constraints are often specified in terms of average current density thresholds or rms [10]. However, having multiple relationships between operating parameters is infeasible. Finally, while the traditional $f-L$ model is characterized at purely capacitive loads, in reality, cells drive $R C$ loads [Fig. 2(b)], and a fast prediction of cell-internal EM safety under $R C$ loads is an open problem.

Our goal is to address four limitations (L1-L4) associated with a chip-level cell-internal EM analysis.

L1: Inability to incorporate the impact of arbitrary switching rates on inputs pins and the effects such as clock gating: we overcome this by discretely characterizing the individual current density components (switching or leakage). In addition, our frequency constraints are selfconsistent, which simultaneously address the average and rms current density criteria, based on formulations proposed in [10].

L2: Inability to comprehend $R C$ loads [Fig. 2(b)] and to model the voltage-dependent pin capacitance $\left(C_{\text {in }}\right)$ : we apply intelligent moment-matching-based techniques as in [3], and propose a novel formulation for $C_{\text {in }}$ estimation.

L3: Inability to retarget reliability specifications on the fly for different reliability conditions: we develop the concept of equivalent stress and present closed-form formulas.

L4: Nonscalability of cell characterization data for an entire library due to prohibitive simulation runtimes, with $\sim 600$ simulations per cell: we perform these simulations efficiently using intelligent response modeling.

The core methodology of our work naturally enables model retargeting by separating the current density computation part from the verification, as against the tight coupling in the model of Fig. 2(a), where the $f-L$ curve must be characterized at each reliability condition. In our approach, the reliability conditions need to be specified in situ: only at the design verification stage. Moreover, our model can take the operating frequency $\left(f_{\text {op }}\right)$ of an instance as an input, or it can provide the maximum safe operating frequency as an output.

\section{EM MODELING-BASIC FRAMEWORK Under PuREly CAPACITIVE LOADS}

\section{A. Electromigration Basics}

In this section, we review the key parameters affecting EM. In our terminology, we refer to metal segments of the IP as resistors. These resistors are obtained by parasitic extraction, which retain key information, such as the width, length, and the metal level for every resistor in the netlist.

Since EM is a statistical process, the time to failure for metal segments stressed in similar conditions also varies [11]. Industrial markets demand low failure rates (e.g., 100 defective parts per million over the chip lifetime). Chip reliability engineers translate this chip-level specification to specific fail-fraction (FF) targets, in units of failures-in-time (FITs), on individual resistors.

The classic Black's equation [11] relates the mean time to failure ( $t_{50}$, time to failure for half of the population) to the average current density $J$ across the interconnect cross section and the wire temperature $T$ as

$$
t_{50}=A J^{-n} e^{Q / k_{B} T} .
$$

Here, $Q$ is the activation energy, $k_{B}$ is Boltzmann's constant, $n$ is the current exponent (typically between 1 to 2 ), and $A$ is a fitting parameter.

Black's equation predicts the time to failure, and in practice, it is predominantly used to determine the average current density thresholds to meet a target FF. It has been demonstrated that FF follows a lognormal dependence on the time to failure ( $t_{f}$, also known as stress time) [11]. The lognormaltransformation parameter $(z)$ relates to the time to failure as 
follows, where $\sigma$ is the standard deviation of the distribution, which is process dependent:

$$
z=\frac{\ln \left(t_{f}\right)-\ln \left(t_{50}\right)}{\sigma} ; \quad \mathrm{FF}=\int_{-\infty}^{z} \frac{e^{-x^{2} / 2}}{\sqrt{2 \pi}} d x .
$$

The transformation variable $z$ helps in directly representing the cumulative failure rate with a normal cumulative distribution function [34]. For example, at stress time $\left(t_{f}\right)=t_{50}, z$ and FF consistently evaluate to 0 and 0.5 , respectively.

In signal wires, currents flow in both directions, leading to a limited damage recovery, which can be incorporated by an empirically estimated recovery factor, $\xi$, used for adjusting the computed average current density in Black's equation [12], as:

$$
J_{\text {avg }}=J_{\text {avg }}^{+}-\xi J_{\text {avg }}^{-} .
$$

Here, $J_{\text {avg }}^{+}$and $J_{\text {avg }}^{-}$indicate the average current density during current conduction in the positive and negative directions, respectively. In addition, the wire heating $(\Delta T)$ has an inherent dependence on the rms current density, $J_{\mathrm{rms}}$, as

$$
\Delta T=c J_{\mathrm{rms}}^{2} \text {. }
$$

Equation (4), with $c$ as a fitting parameter, follows directly from heat conduction principles. Typically, the limit on the maximum temperature rise due to $\mathrm{JH}$ is a design constraint, and this place automatically limits on rms current densities.

Pioneering work in [10] combined the two effects of average EM fails and rms-induced $\mathrm{JH}$ in a self-consistent manner through the concept of duty cycles, making it possible to simultaneously check both conditions. Thus, given the constraints of stress temperature, lifetime, and JH limit, we can arrive at the EM thresholds that should be met by all metal segments in the IP. Once we have the EM thresholds in place, we can embark on the EM verification process across various resistors in the IP.

It must, however, be noted that fundamentally, EM is induced by divergence of atomic flux-which is typically highest at sites, such as vias, contacts, or even points, where the leads merge. Furthermore, it has been reported in the literature that even if the incoming atomic flux (signified by high current density) is high at such sites, the site itself may not fail due to it maintaining a low divergence; while a simple, individual-lead-based Black's equation continues to predict failure for such a structure. This inefficiency has recently been revisited by various researchers resulting into the evolution of alternate paradigms in EM checking [31]-[33]. Fundamentally, such alternative methods rely on computing some form of atomic flux divergence at EM-probable sites and, subsequently, comparing them against set thresholds. One such method, as reported in [31], is the vector via-node-based method, wherein the physical and directional interactions among various leads are incorporated to perform the reliability verification. Notably, however, the fundamental inputs required to perform these calculations still remain the individual current density in every single interconnect of the circuit, along with the additional information like the circuit topology.

Consequently, we note that even for alternative EM checking methodologies, the discrete current density in individual interconnects is still the vital input-which is discussed in more detail in Section III.

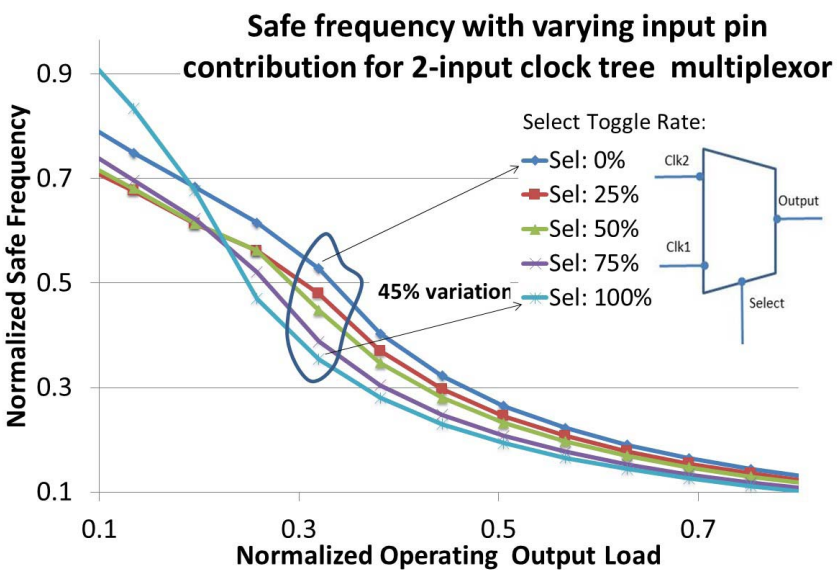

Fig. 3. $f_{\text {safe }}$ plot for a two-input clock-multiplexor cell. Both input clocks switch at $100 \%$, while the select pin chooses one of them, with varying likelihoods.

\section{B. Traditional Approach for Modeling EM Reliability}

We begin by revisiting the traditional approach, as shown in Fig. 2(a). Given the physical design of the IP, EM verification requires a model that provides a tradeoff among various operating conditions such that within the bounds of those tradeoffs, the IP remains EM-safe. The generation of this model requires an iterative search: for example, in Fig. 2(a), at a fixed loading and reliability condition (say, $50 \mathrm{fF}, 1 \mathrm{~V}$, $\left.105{ }^{\circ} \mathrm{C}, 100 \mathrm{k} \mathrm{POH}\right)$, an iterative search over the frequency space is required to determine the maximum $f_{\text {safe }}$, where all resistors within the IP are EM-safe. This is computationally expensive, since each iteration involves an SPICE-simulationbased verification. A typical optimized procedure requires ten binary-search iterations at each loading condition. For a single-input cell, whose operating load/slew space is covered through an $8 \times 8$ matrix in the liberty file, the number of required iterations is $\sim 64 \times 10=640$ for fixed values of other parameters (supply voltage and reliability specifications). To support operation at multiple supply voltages, as well as IP reuse across application domains, this number must be multiplied by the number of use cases, resulting in a formidable characterization overhead.

While this may even be tractable for single-input cells, for multiple-input cells, this characterization becomes challenging, not only just from a computational point of view, but also from the fundamental modeling (L1) viewpoint. To illustrate this idea, we consider the example of a two-input clocktree mux IP block that is used to alternate among clocks for downstream propagation. The user may examine typical workloads, use cases, and provide an EM analysis tool with information about the switching rates of the input pins of a block. In this experiment, both the input pins (Clk1, Clk2) switch at $100 \%$, but the select pin is toggled to allow passing of the first and second clocks in varying amounts (going from $0 \%$ to $100 \%$ in steps of $25 \%$ ).

The $f-L$ plots for the five cases are shown in Fig. 3, and show a variation of up to $45 \%$ in $f_{\text {safe }}$ estimates, depending on how often Clk1 or Clk2 is selected over the lifetime, but the traditional model will choose the pessimistic $f_{\text {safe }}$ over all cases. We also see that while at very low loads, select $=100 \%$ (meaning Clk2 being transmitted) results in least EM stress, it switches over as the load increases. This switch results 
from the interplay of various currents (short-circuit and the output switching current) in the circuit. Such an asymmetric response can only be captured by the traditional model by individually generating and storing the $f-L$ data for various input excitations, which is computationally expensive. Further, effects like clock gating are not straightforward to handle in the traditional model.

Another significant drawback (L2) with the traditional model is the fact that it has been generated using a lumped $C$ load, while real applications involve $R C$ loading. Due to resistive shielding effects, a direct application of the traditional model to assess reliability of instances that drive $R C$ loads turns out to be severely pessimistic. Finally, we also note that the traditional model is locked to a particular reliability specification (supply voltage, temperature, lifetime, and failure rate target), and is incapable in allowing a tradeoff on these (L3), unless, the $f-L$ data are regenerated along these vectors, which becomes computationally unaffordable for the entire library (L4). With the above background and detailed understanding of the traditional model (including generation, usage, and associated limitations), we now look at building the proposed model, which can address the various limitations.

\section{AdDRESSING L1-INCORPORATING ARBITRARY SWITCHING AND ClOCK GATING IN FREQUENCY ESTIMATION}

\section{A. Library Level Current Density Characterization}

In order to build the model that can help predict the reliability of an IP for arbitrary switching scenarios, we begin in an $a b$ initio manner by trying to classify the current flow in the IP as either leakage or switching current. We observe that for a combinational IP with $m$ inputs, $2^{m}$ distinct static states (various combinations of input pins at logic 1 or 0 ) are possible. Each of these states can have a different leakage flow. In addition, based on the IP functionality, there could be several paths (later referred to as arcs) from an input pin resulting in an output transition. Every such output transition causes a switching current flow in the IP-internal resistors (belonging to the resistor-set $\Re$ ).

Thus, the first step in our approach is to discretely characterize the current flow: average and rms, both through every resistor $R$ in the IP (resistor-set $\Re$ ), in every legal state (for leakage current), or arc through the cell (for switching current). Such a characterization will be used to compute the eventual effective current density through any resistor of the cell as a weighted summation of the current densities in unique scenarios, coupled with the information of arc switching rates and probabilities of legal state occurrences.

The salient feature of our characterization is that it remains independent of the reliability condition, which is actually an input during chip-level verification. As the leakage current density in the cell only depends on the static states of inputs, we can easily obtain the current density through $\Re$ by cycling through all possible input states in SPICE (note that average and rms remain the same due to dc nature of the waveform). On the other hand, switching current densities are tied to a particular input-pin to output-pin combination (also referred to as a timing arc), through a fixed cell-internal path, with other inputs in noncontrolling states enabling the transition. For example, for a three-input AOI gate $(Y=!(A+B C))$, the output $Y$ can fall because of a rise on $A$ in three different states of $B C$, namely, 00,01 , and 10 . Hence, for this particular $A \rightarrow Y$ arc, the current density must be computed through $R$ for these three states of $B C$. We can leverage the simulation framework of industrial timing characterization systems [9], to obtain information about all such arcs and states through the cell. For a particular arc $i$ and associated noncontrolling state $k$, we denote the time duration over which this current density is calculated as $s_{i k}$. A similar convention is followed by $J_{\text {avg }, R_{i k}}$ and $J_{\mathrm{rms}, R_{i k}}$ to define the average and rms current densities through $R$. As we leverage the timing characterization framework, we do not recompute $s_{i k}$, but reuse it from the timing analysis step [8], [13]. Moreover, $s_{i k}$ is typically greater than the delay itself and, therefore, accurately captures the tail effects.

\section{B. Effective Current Density Estimation for a Chip-Level Instance}

After characterizing the leakage and switching current densities for various arcs and states, we now present the calculations for the effective average and rms densities in the circuit.

1) Effective Leakage Current Density Through a Resistor Across All States: For an $m$-input gate, let the leakage current density through resistor $R$ for a state $k$ (of $2^{m}$ states) in the positive (negative) direction be denoted by $L_{+R_{k}}\left[L_{-R_{k}}\right]$. Then, the average effective leakage current density $\left(L_{\mathrm{avg}, R}\right)$ covering all the states and incorporating recovery (3) would be

$$
L_{\mathrm{avg}, R}=\sum_{k=1}^{l} P_{k}^{+} L_{+R_{k}}-\xi\left(\sum_{i=1}^{2^{m}-l} P_{k}^{-} L_{-R_{i}}\right) .
$$

Here, $l$ is the number of states with a positive current density, and $P_{q}^{+}\left[P_{q}^{-}\right]$is the probability of occurrence of state $q$, in which the current flows in the positive (negative) direction. These probabilities are a function of the duty cycle at the inputs of the gate.

The rms effective leakage current density is given by

$$
L_{\mathrm{rms}, R}^{2}=\sum_{k=1}^{l} P_{k}^{+} L_{+R_{k}}^{2}+\sum_{i=1}^{2^{m}-l} P_{k}^{-} L_{-R_{k}}^{2} .
$$

2) Effective Switching Current Density Through a Resistor Across All Switching Arcs: In similar spirit, the effectiveaverage-switching current density through $R\left(J_{\mathrm{avg}_{\mathrm{sw}}, R}\right)$ is given by

$$
J_{\mathrm{avg}_{\mathrm{sw}}, R}=\sum_{i=1}^{\text {all arcs }}\left(\sum_{k=1}^{\text {all states }} P_{i k} J_{\mathrm{avg}, R_{i k}} \frac{s_{i k}}{T_{\mathrm{clk}}}\right) .
$$

Here, $P_{i k}$ and $T_{\mathrm{clk}}$ are the design-level parameters-the switching probability of the particular arc and the switching period, respectively. The scaling factor, $s_{i k} / T_{\text {clk }}$, translates the characterized current density $\left(J_{\mathrm{avg}, R_{i k}}\right)$, which was averaged during the characterization over the switching duration $s_{i k}$, into the entire clock period. This scaling factor accounts for the fact that the current is inactive during the remainder of the clock period. Similar calculations for rms current density $\left(J_{\mathrm{rms}_{\mathrm{sw}}, R}\right)$ yield

$$
J_{\mathrm{rms}_{\mathrm{sw}}, R}=\sqrt{\sum_{i=1}^{\text {all arcs }}\left(\sum_{k=1}^{\text {all states }} P_{i k} J_{\mathrm{rms}, R_{i k}}^{2} \frac{s_{i k}}{T_{\mathrm{clk}}}\right)} .
$$


3) Effective Average and RMS Current Densities: After computing the effective switching and leakage current densities independently, we must now compute the effective average and rms current densities. In a normal design flow, the chiplevel probabilistic activity propagation tools already provide the effective switching rate $\left[f_{i k}=\left(P_{i k} / T_{\mathrm{clk}}\right)\right]$ for any given arc $i$ and associated noncontrolling state $k$, along with the state probabilities $\left[P_{q}^{+}\right.$in (5a)] for all gates of the design.

Since (5)-(7) discretely describe the leakage and switching current densities, we can sum them to derive the effective average current density $\left(J_{\mathrm{avg}, R}\right)$ and add them in an rms manner to derive the effective rms current density $\left(J_{\mathrm{rms}, R}\right)$ for any resistor $R$ in the cell.

We compute the average and rms current densities by consolidating (5)-(7) as

$$
\begin{aligned}
& J_{\mathrm{avg}, R}=J_{\mathrm{avg}}, R+L_{\mathrm{avg}, R} \\
& J_{\mathrm{rms}, R}^{2}=J_{\mathrm{rms}, \mathrm{sw}, R}^{2}+L_{\mathrm{rms}, R}^{2} \text {. }
\end{aligned}
$$

It must be mentioned here that rms formulations work under the assumption that the different current densities (leakage and switching) are nonoverlapping. This strictly is not true; however, we find that this assumption leads to very marginal errors. Next, we look at incorporating clock gating in the formulations.

4) Incorporating Clock Gating: Clock gating is a widely used technique for reducing the dynamic clock power by disabling the clock signal to the idle parts of the circuitthereby also directly affecting the reliability of the signals in the gated domain [28]. In order to assess the reliability impact of clock gating, we notice that as a phenomenon, clock gating can occur in an arbitrary way over the lifetime of the chip. For instance, the clock could be gated for a fixed number of cycles, after every specific period of activity, in a repeated manner. Such uniform gating is akin to a direct reduction in the operating frequency and can be readily approximated by specifying the activity-rate-adjusted frequency in (8) and (9).

However, the cases when the clock gating is nonuniform, or is uniform only in the intervals, are nontrivial and require equivalent reliability-lifetime calculations. The key determinant in such calculations is the thermal time constant of $\mathrm{JH}$ in interconnect (typically in several microseconds for copper [30]), which signifies the duration after which the interconnect responds to the rms current in the form of a temperature rise. Hence, if the time interval between successive clock gating events is larger than the thermal time constant, then the full current (without activity correction) should be ideally used for rms and average density estimations, for the appropriate durations.

We will defer the treatment for nonuniform clock gating to Section V-B (subsequent to incorporation of arbitrary reliability specifications), and only focus the formulations now for the uniform case. This makes the solution similar to setting a pin specific activity rate on the cell. Hence, if a $1-\mathrm{GHz}$ clock-tree element remains gated-high for $25 \%$ of the lifetime, we would note the corrected $f_{i k}$ as $750 \mathrm{MHz}$ in (8) and (9), and state probability as 0.375 (assuming 50\% duty cycle for clock). The computation procedure can thus be captured as follows.

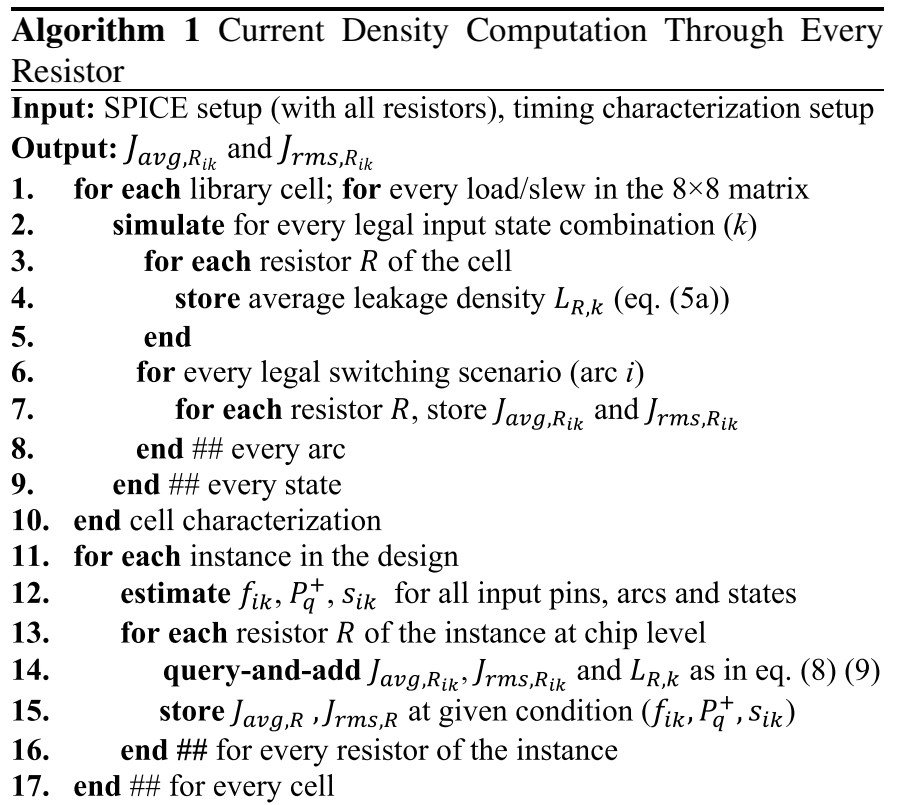

\section{Instance Safe Frequency Estimation at Chip Level}

Once we have estimated the current densities in the cell, the EM checking procedure can subsequently be approached in two manners, as noted in Section II earlier.

1) Predict the safety of the cell (pass or fail), given a full set of operating conditions of the cell.

2) Calculate a set of safe operating parameters for the cell under a partial set of operating conditions. For example, if the frequency, slew, and supply voltage are given, the safe load may be computed.

The first is rather trivially obtained from the above discussion, since (8), (9), and Algorithm 1 lend themselves readily to allow substitution of the exact operating conditions, and subsequent verification of current densities (through all resistors) against the foundry EM thresholds.

In real designs, however, the actual operating frequency of the instance could be arbitrary, and we must work the problem backwards by recommending a maximum $f_{\text {safe }}$ based on other parameters. In contrast to the $f-L$ data of Fig. 2(a) obtained by iterated binary-search SPICE simulations, our approach here provides closed-form solutions for $f_{\text {safe }}$.

It must be noted that potentially, every resistor in the cell could have a unique frequency dependence, and therefore, the maximum $f_{\text {safe }}$ procedure must find the minimum safe frequency over all resistors in the instance.

Let $J_{\mathrm{avg}, \mathrm{th}}(T, t)$ and $J_{\mathrm{rms}, \text { th }}(\Delta T)$ represent the current density limits for average and rms current densities, respectively, as a function of stress temperature, stress time, and maximum heating constraint. Furthermore, note that in (5)-(7), the dependence on the frequency $f=1 / T_{\text {clk }}$ only appears in the expressions for the average and rms switching current densities. By setting the left-hand sides of (8) and (9) to be no larger than the threshold densities and combining them with (5)-(7), we can constrain the rms or average-limited frequencies ( $f_{\max , \mathrm{AVG}, R}$ and $f_{\mathrm{max}, \mathrm{rms}, R}$, respectively) for each 


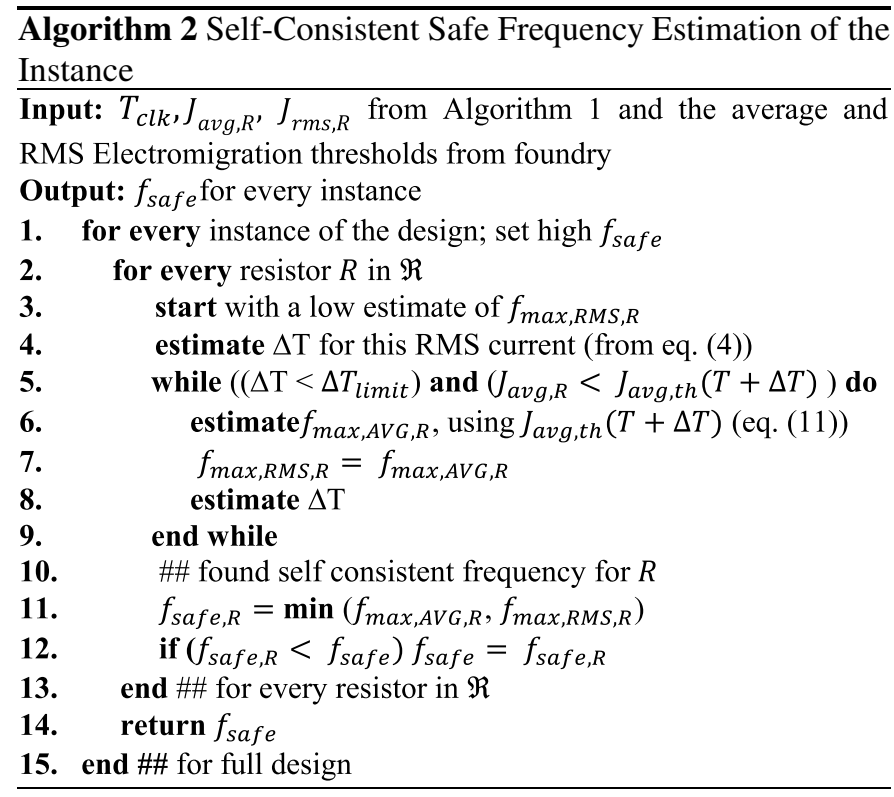

intracell resistor $R$ in the following manner:

$$
\begin{aligned}
& f_{\mathrm{max}, \mathrm{AVG}, R}=\frac{J_{\mathrm{avg}, \mathrm{th}}(T, t)-L_{\mathrm{avg}, R}}{\sum_{i=1}^{\text {all arcs }}\left(\sum_{k=1}^{\text {all states }} P_{i k} J_{\mathrm{avg}, R_{i k}} s_{i k}\right)} \\
& f_{\mathrm{max}, \mathrm{RMS}, R}=\frac{J_{\mathrm{rms}, \mathrm{th}}^{2}(\Delta T)-L_{\mathrm{rms}, R}^{2}}{\sum_{i=1}^{\text {all arcs }}\left(\sum_{k=1}^{\text {all states }} P_{i k} J_{\mathrm{rms}, R_{i k}}^{2} s_{i k}\right)} .
\end{aligned}
$$

Since all parameters on the right-hand sides of the above equations are known for each resistor in each instance, we can now apply the self-consistent formulations [10] to estimate the safe parameter (frequency) of the resistor. The entire process has to be approached iteratively, as shown in Algorithm 2, to determine the safe operating frequency for an instance, which can then be used as a design constraint. The safe frequency for a resistor is the lower of the two values in (10) and (11), and the safe frequency $f_{\text {safe }}$ for a cell instance is the smallest safe frequency over all resistors in the instance.

To evaluate this procedure, we revisit the two-input clock-tree mux from the earlier discussion around Fig. 3. Fig. 4 shows the $f_{\text {safe }}$ plot for this case, for a fixed operating condition and output load, showing the results of the binary-search-based SPICE simulation, our approach, and the traditional method that chooses $f_{\text {safe }}$ pessimistically over all switching conditions. We see that the proposed model fits the SPICE behavior very well and can model the arbitrary switching rates on different pins, as against the large pessimism in the traditional approach.

While the results shared in Fig. 4 were from a single cell, consolidated results from the entire 28-nm design library will be shared later in Section VII. It must also be noted that thus far, we have demonstrated Black's equation (1)-based EM verification. However, as our methodology aptly decouples the current density computation and the verification part, it easily lends itself to other EM verification schemes, such as the via-node-based scheme (Section II-A).

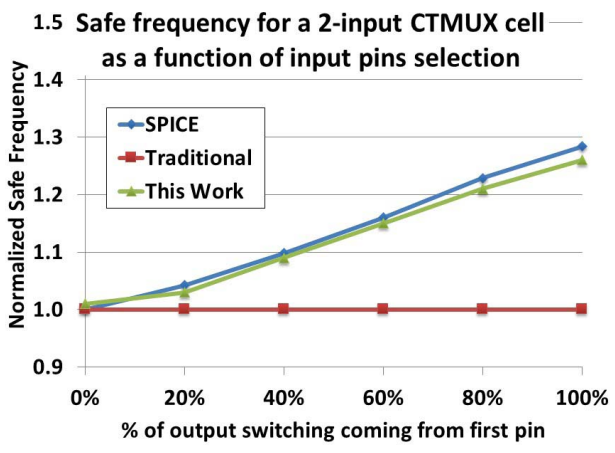

Fig. 4. Evaluation of $f_{\text {safe }}$ for the circuit in Fig. 3, at a selected load point: $0.45 \times$. The value of $f_{\text {safe }}$ varies based on the extent of switching coming from the first or second pin. The proposed model completely captures the behavior, but the traditional is excessively pessimistic.

\section{AdDRESSING L2: MODELING THE IMPACT OF ARBITRARY $R C$ LOADING}

The model developed so far is capable of covering following parameters: lumped capacitive load ( $C$ load), slews, multi-input gates, arbitrary switching rate, and clock gating. This is directly relevant at the chip level, when the IPs are used at arbitrary frequencies and under clock gating. Next, we look at incorporating $R C$ load into the assessment.

\section{A. Overview of Prior Work}

In Section III, we used the lumped load as one of the metrics for EM reliability. To a great extent, the $C$ load model in itself can be used for accurate estimation of average current density, and is largely independent of resistive effects [3], provided the rail-to-rail swings for the output net.

On the other hand, RMS current densities additionally depend on the duration of transfer, and are thereby directly impacted by the resistive effects of RC loads on the cell [14][19]. The effect of RC load [Fig. 2(b)] for signal EM reliability was addressed earlier in [3]. It was further established that resistive shielding cannot be accounted using the traditional $C_{\text {eff }}$ approach, derived from timing constraints. Hence, a current-criterion-based moment matching was devised to come up with a $C_{\text {eff }}$, by performing the $\mathrm{RC}$ tree traversal along with the basic timing information.

\section{B. Prior Work: Limitations}

We notice that there are at least two limitations of the prior work associated with both the traditional model [Fig. 2(a)] and the model proposed in Section III.

First, $R C$ loads affect the current flow in all segments: cell-external and cell-internal. While the cell-external problem was solved in [3], the cell-internal piece of the problem has remained unsolved. In fact, it was proposed to simulate the entire active network with the actual distributed load (at transistor level) through SPICE. As we will see later (Section VII), the number of such simulations required for a block/SoC could run in thousands, becoming a major computational and logistical overhead.

Second, we notice that not just the effective capacitance, but also the lumped capacitance depends on the current waveform shape-making the load capacitance itself as voltagedependent. This can be explained by the fact that the pin capacitance has an inherent voltage dependence [20]. Hence, 


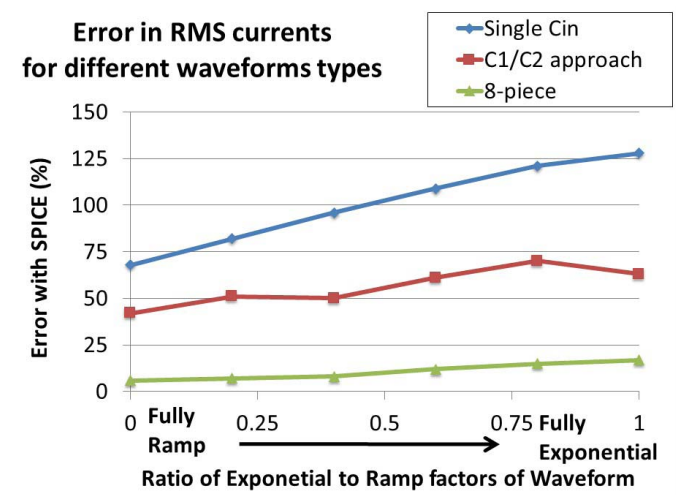

Fig. 5. Error in the rms estimates (versus SPICE) for various $C_{\text {in }}$ modeling approaches and waveform types ( $x$-axis; going from fully ramp to fully exponential).

even though there is no explicit dependence of the average current on the network resistance [3], it implicitly exists because of the dependence on $C_{\mathrm{in}}(V)$. Therefore, assuming a fixed value of $C_{\text {in }}$ for performing current density calculations on a net becomes very pessimistic. We now attempt to solve both problems.

\section{Proposed Solution: $R C$ Loading and $C_{i n}$ Modeling}

We begin by observing that the basic challenge for cellinternal EM arises from the fact that the characterization of the fundamental current densities (through Algorithm 1) must be performed using the single $C$ load values, but the data must be applied to instances that drive $R C$ loading. Hence, we require a good proxy of the $R C$ load, which can be used to query the characterized data. Extending the concepts developed in [3], if we use $C_{\text {eff }}$ to query only the RMS component of the current density from the precharacterized data, an accurate match can be achieved. Indeed, we do see a reduction in error (compared with SPICE) in this manner: as we will later see in Fig. 6, the mean error of about $2 \mathrm{X}$ in RMS estimation reduces to about $20 \%$ with the $C_{\text {eff }}$ incorporation. However, there still are outliers and upon detailed investigation, a majority of them are attributable to $C_{\text {in }}$ modelingof the load pins.

Next, as an improvement, we also compared the current densities derived from the case, when the load cells were modeled as a $C 1 / C 2$ combination, where $C 1$ represents the pin capacitance from $0 \%-50 \%$ swing of the voltage, and $C 2$ from $50 \%-70 \%$ [21]. However, we notice that at an individual load pin level itself, this approach does not yield high accuracy due to ignorance of the tail effects [3], [22].

Hence, we propose calculating an effective $C_{\text {in }}\left(C_{\text {in,eff }}\right)$ from the multipiece $C_{\text {in }}(V)$ table [Fig. 5; typically eight points]. Since $C_{\mathrm{in}}$ is a function of the voltage waveform, which, in turn, is a function of $C_{\mathrm{in}}$, the entire computation must be carried out in an iterative manner. Accordingly, in the $k$ th iteration, we make use of the starting current waveform [as incident on the load cell $L_{i}$ of Fig. 2(b)]. Such a current waveform $\left(I_{L_{i, k}}(t)\right)$ is obtained through a single $C_{\mathrm{in}, k}$ and uses a double exponential model with estimated parameters- $A_{0, k}$, $T_{a, k}$, and $T_{b, k}$. The estimation of these parameters is performed by $R C$-tree traversal and moment matching technique with assumption on the waveform shape at the driver (a mixture of ramp/exponential) [3]. The current waveform is modeled as

$$
I_{L_{i, k}}(t)=A_{0, k}\left(e^{-t / T_{a, k}}-e^{-t / T_{b, k}}\right) .
$$

Subsequently, the voltage waveform $V_{L_{i, k}}(t)$, as seen on the load pin, can be generated as an area under the curve of this current waveform, using a constant $C_{\mathrm{in}, k}$

$$
V_{L_{i, k}}(t)=\frac{1}{C_{\mathrm{in}, k}} \int_{0}^{t} I_{L_{i, k}}\left(t^{\prime}\right) d t^{\prime} .
$$

This voltage waveform can then be used along with the varying $C_{\text {in }}: C_{\text {in }}(V)$ table to reconstruct a new current waveform $I_{L_{i, k}}^{\prime}(\mathrm{t})$ as

$$
I_{L_{i, k}}^{\prime}(t)=C_{\text {in }}(V) \frac{d}{d t}\left(V_{L_{i, k}}(t)\right) .
$$

Note that only an update in the current waveform at the load pin is required, since we are interested in the current specifically at this point. Assuming the duration of this current waveform as $d$ (approximated by the corresponding 0\%-100\% slew at the load pin obtained through STA), its rms is given by

$$
\text { RMS for } I_{L_{i, k}}^{\prime}(t)=\sqrt{\frac{1}{d} \int_{0}^{d} I_{L_{i, k}}^{\prime 2}(t) d t} \text {. }
$$

Note that for the next iteration, we require an updated value for $C_{\text {in }}$. Hence, we make use of the rms current through $I_{L_{i, k}}^{\prime}(\mathrm{t})$, to derive a single effective $C_{\text {in }}$, assuming an equivalent triangular current waveform (with $d$ being the delay at the load pin). For such a triangular waveform, the rms current expression is standard: $\sqrt{(4 / 3)}(C V / d)$, where $C$ is the equivalent load. In order to obtain an equivalent pin capacitance that can match the rms current of $I_{L_{i, k}}^{\prime}(\mathrm{t})$, we equate (15) to the rms current of triangular waveform, to get below capacitance (to be used for the next iteration) as

$$
C_{\mathrm{in}, k+1}=\frac{d}{V} \sqrt{\frac{3}{4}} \int_{0}^{d} I_{L_{i, k}}^{\prime 2}(t) d t .
$$

We expect convergence in 2-3 iterations, though, for our work, we have made only a single update to starting $C_{\text {in }}$. In similar way, the average current case can be approached. While this means that we must ideally compute two separate capacitances: namely $C_{\text {in,eff,rms }}$ and $C_{\text {in,eff,AVG }}$ our experiments indicate acceptable errors for the average case, and hence we do iterative computation only for RMS matching.

In summary, we accurately incorporate the impact of the voltage-dependent input-pin capacitance as well as the impact of parasitic $R C$ loading on the cell-internal current densities by the following.

1) Making an initial estimate of the current at the driving points and iterating with the load's voltage-dependent pin capacitance to arrive at the final current flow.

2) Estimating the effective capacitance ( $\left.C_{\text {eff }}\right)$, which matches the final current flow in the network.

3) Using this $C_{\text {eff }}$ to query the precharacterized cell-internal rms current density database and $C$ load for querying AVG cell-internal current densities.

Note that in absence of this method, we would have used $C$ load to query the cell-internal AVG as well as rms current densities, which is very pessimistic. Note also that the formulations for incorporating voltage-dependent pin capacitance automatically improve the accuracy of cell-external current densities as well. 


\section{D. $R C$ Loading and $C_{i n}$ Model Validation: Results}

We perform validation at multiple levels of the $R C$ and $C_{\text {in }}$ modeling approaches. First, we validate the $C_{\mathrm{in}}$ approach at the load-level circuit, followed by the validation of the combined $R C$ loading and $C_{\text {in }}$ modeling in the driver-load pair case [Fig. 2(b)] and followed finally by the results from several driver instances (driving unique $R C$ loads).

We begin by showing the load-level comparison first, for effective $C_{\text {in }}$ estimation (versus SPICE) for different $C_{\text {in }}$ models [Fig. 5]. This comparison is at the load circuit level, where we apply a voltage waveform at the load pin and the load cell is modeled as: 1) single $C_{\text {in }}$;2) a two-piece voltage-dependent capacitor in SPICE [23]; and 3) a single $C_{\text {in,eff }}$ [obtained from (12)-(16)].

Moreover, as discussed earlier, since $C_{\text {in }}$ is a function of the starting input voltage waveform, we have computed the errors for different types of input voltage waveforms (the $x$-axis represents waveforms going from fully ramp to fully exponential), whose shape is controlled with the coefficient $a$ in below equation, with $T_{r}$ being the rise time

$$
V_{\text {in }}(t)= \begin{cases}a\left(1-e^{-t / T_{r}}\right)+(1-a) \frac{t}{T_{r}}, & t \leq T_{r} \\ 1-a e^{-t / T_{r}}, & t>T_{r} .\end{cases}
$$

Hence, setting $a$ to zero in (17) results in a fully saturated ramp input waveform, whereas setting $a$ to unity makes it complete exponential. Such a formulation is a good representation of the various input waveforms that can be incident on the load pin.

As we can see from Fig. 5, the traditional approach of single $C_{\text {in }}$ leads to almost $2 \times$ error as compared to SPICE. The error reduces using the $C 1 / C 2$ model, but it still remains unacceptable, and the effective capacitance computation approach from an eight-piece piecewise-linear table fits the SPICE results in a better solution. We can also see that because of increased tail effects in the exponential input voltage waveform, the errors are higher for all models for a completely exponential case.

Fig. 6 shows the maximum error from several instances (which drive different $R C$ loads; plotted on the $x$-axis). While the left $y$-axis shows the errors, the $C_{\text {eff }} / C$-load ratio, an indicative of the extent of resistive load the instance is driving, is plotted on the right $y$-axis. The exact set of instances and their driving $R C$ load information are obtained from a 28 -nm production design. We show the comparison of the traditional case (using lumped load for current density querying), $C_{\text {eff }}$ model alone and the combined $C_{\text {eff }}+C_{\text {in }}$ model.

Overall, among all cases, we find about $2 \times$ mean error in rms current density estimation with the usage of lumped load, which drops to $\sim 21 \%$ mean with the usage of $C_{\text {eff }}$ model, and further down to $\sim 7 \%$ mean error with the combined usage of $C_{\text {eff }}$ and $C_{\text {in }}$ model. We also see that for instances, driving severely resistive loads (indicated by the ratio of $C_{\text {eff }}$ to $C$ load), the original error with $C$ load usage is very high, with outliers that cross $50 \%$ error.

Algorithm 3 shows the final procedure for estimating the accurate cell-internal currents for arbitrary loading.

Thus, we have examined the impact of $R C$ loading on the EM reliability, and demonstrated significant improvement in accuracy with the proposed method.

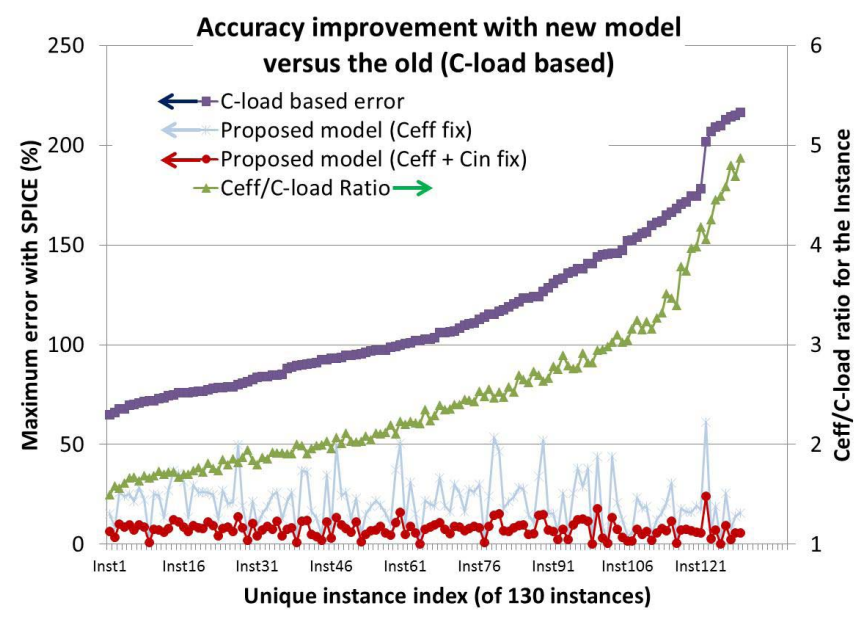

Fig. 6. Maximum error in rms current density estimation across several instances driving different kinds of $R C$ loading (indicated by the $C_{\text {eff }} / C$-load ratio) at the design level.

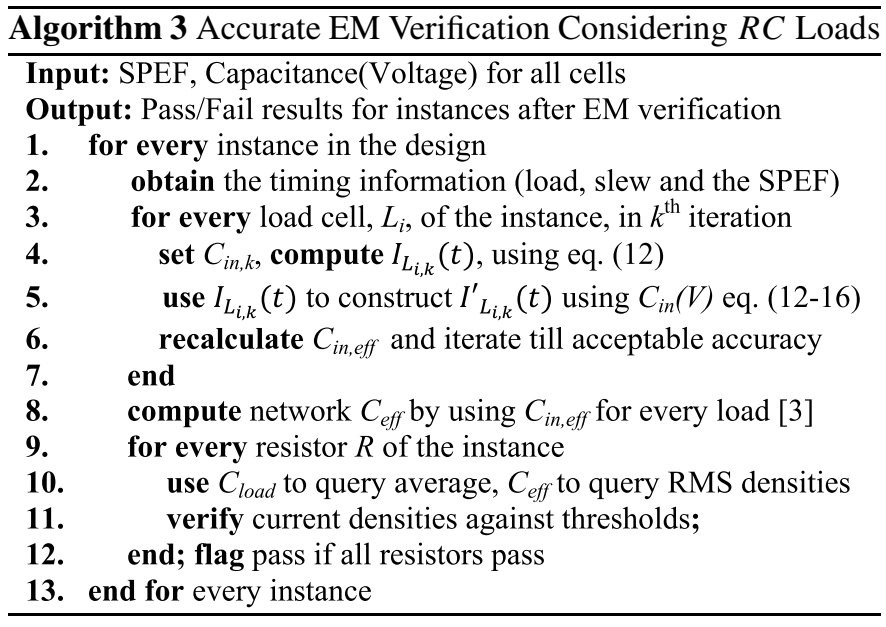

\section{AdDRESSing L3-ON-THE-Fly RetARgETING OF RELIABILITY FOR ARBITRARY SPECIFICATIONS}

The formulations of Sections III and IV were dependent on the library data characterized at one set of operating conditions, and the foundry EM thresholds at a specified reliability condition. However, as described in Section I, there is an increasing need for on-the-fly reliability retargeting, at design verification stage, as the IP library is used under different reliability conditions. As noted earlier, meeting this goal is impractical under the traditional methodology, as it requires a new characterizations of the entire IP library [Fig. 2(a)] at each new condition.

The core methodology of this paper enables the ability to perform this retargeting efficiently, since the current density computation part is separated out from the verification part (whereas these are tightly coupled in the traditional approach). We begin with the fundamental relation between the EM lifetime and the lognormal variable. From (1) and (2), taking logarithm, we obtain

$$
\sigma z=\ln \left(t_{f}\right)-\ln (A)+n \ln (J)-\frac{Q}{k_{B} T} .
$$

Now, if we have two different sets of stresses, denoted by subscripts $a$ and $b$, each is described by the same fitting 
parameter $A$, but other terms in (18) may differ. Naturally, their reliability is related as follows (by substituting the parameter $A$ ):

$$
\begin{aligned}
\sigma z_{\text {cond }, b}= & \sigma z_{\text {cond }, a}+\ln \left(\frac{t_{b} J_{b}^{n}}{t_{a} J_{a}^{n}}\right) \\
& -\frac{Q}{k_{B}}\left(\frac{1}{T_{b}+\Delta T_{b}}-\frac{1}{T_{a}+\Delta T_{a}}\right) .
\end{aligned}
$$

Here, the variables $t, J, T$, and $\Delta T$ represent the stress time, current densities, stress temperature, and $\mathrm{JH}$, respectively, while the subscripts $a$ and $b$ refer to the two different conditions.

This equation is a powerful representation of the scaling factors that can either be used to assess: 1) the required tradeoffs in new reliability conditions to meet the same FF levels or 2 ) the actual FFs at the new reliability conditions. For example, we can directly use above equation to find the equivalent stress time $\left(t_{b}\right)$ that causes the same reliability loss as benchmark condition, but with increased current densities. In order to do so, we must set $z_{\text {cond, } b}=z_{\text {cond, } a}$, since the reliability loss has to be equated and obtain the equivalent lifetime $t_{b}$ as a function of $\left(t_{a}, J_{a}\right.$, and $\left.J_{b}\right)$. Obviously, if $J_{b}>J_{a}, t_{b}$ will be estimated to be lower than $t_{a}$.

We now look at the application of the retargeting concepts, based on (19), to some of the case studies, followed by application to nonuniform clock gating. Unlike uniform clock gating, which was previously treated with generic activity reductions in Section III-B, nonuniform clock gating requires a more accurate sliding window-based analysis, wherein every frame potentially becomes a new reliability condition.

\section{A. Case Studies Incorporating Reliability Retargeting}

1) Case I (Variations in Temperature): If the use temperature and/or $\mathrm{POH}$ specification are different from the original conditions, then it is straightforward to address this by using (19) to determine new current density thresholds, and then updating $f_{\text {safe }}$ in (11). Such a modification only affects the average, and not the rms reliability.

A second situation is the common industry scenario when the stress profile is provided by the user as a temperature profile, as the series $\left\{\left(J_{1}, T_{1}, t_{1}\right),\left(J_{2}, T_{2}, t_{2}\right), \ldots,\left(J_{m}, T_{m}, t_{m}\right)\right\}$, i.e., from time $t_{k-1}$ to time $t_{k}$, it experiences current stress $J_{k}$ at temperature $T_{k}$. If the baseline stress is characterized for $J_{0}$ at temperature $T_{0}$, then it can relate the $k^{\text {th }}$ stress vector to the baseline stress at $\left(J_{0}, T_{0}\right)$ with an equivalent stress time $t_{k, 0}$. In other words, the stress at temperature $T_{k}$ is transposed to an equivalent stress time at temperature $T_{0}$. Consequently, our stress retargeting scheme will map the entire stress to $\left(J_{0}, T_{0}, t_{\text {eq }, 0}\right)$, where $t_{\text {eq }, 0}=\sum_{k=1}^{m} t_{k, 0}$.

2) Case II (Variation in Operating Voltage): If the eventual use voltage of the library is different from the characterization voltage, current scaling must be performed. Such a scaling is straightforward in our framework, since the leakage and switching related components are separately stored, as described in (6) and (7). Based on our experiments, we see that a linear scaling works very well for voltage scaling, while an exponential model is required for leakage. Note that this scaling must be performed for every discrete component of the current densities for every resistor in the circuit (6), (7).

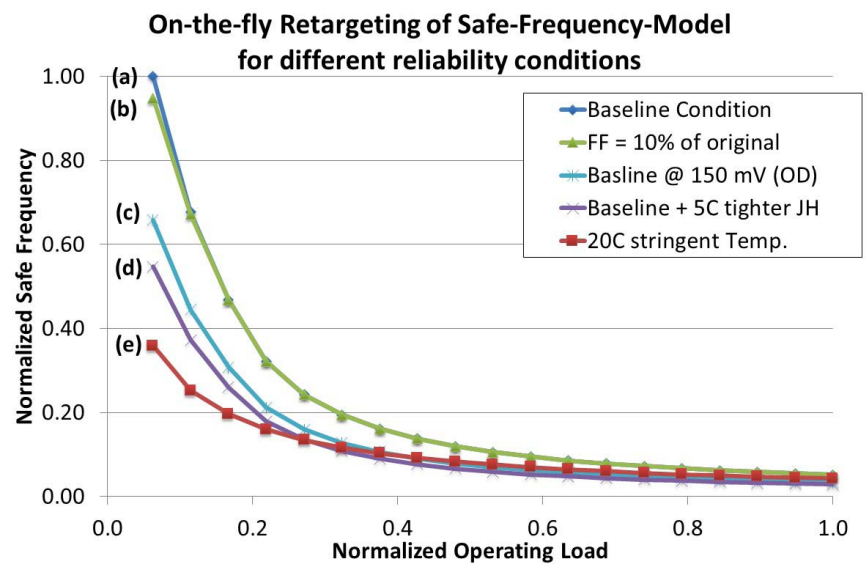

Fig. 7. Demonstrating on-the-fly retargeting of the basic frequency-load curve [Fig. 2(a)] with changes in the constraining criteria (at a fixed slew point).

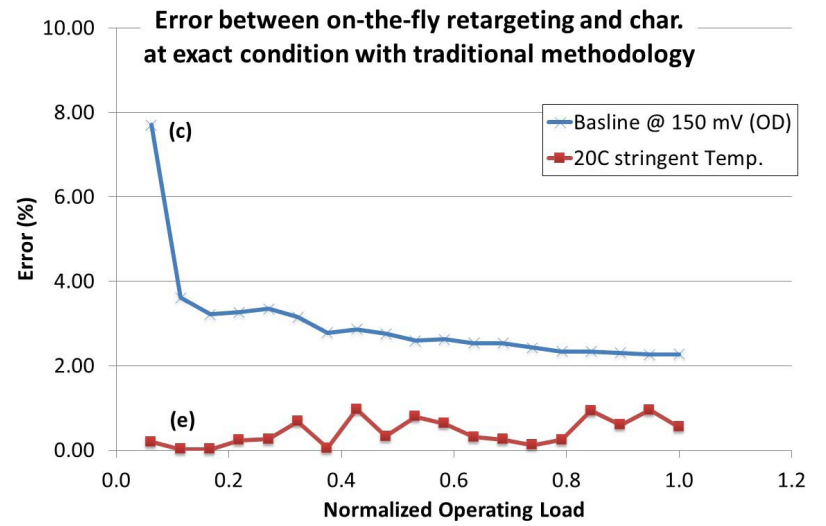

Fig. 8. Validation of retargeting methodology versus SPICE for two conditions, curves c) and e), of Fig. 7.

A second situation (arising due to power management scenarios like dynamic voltage frequency scaling [24]) is when the voltage is represented as a series: $\left\{\left(V_{1}, t_{1}\right) \ldots\left(\left(V_{m}, t_{m}\right)\right\}\right.$. In such a case, we can follow the scaling procedure to obtain a series of currents, which can then be dealt in the same way as the earlier case.

3) Case III (Variation in Failure Rate Specification): $J_{\text {avg,th }}(T, t)$ in (11) is really a function of the FF, which, in turn, is a function of $z$ (2). Therefore, $z_{\text {target }}$ is the inverse function of $\mathrm{FF}_{\text {target }}$. Hence, if the FF specified by the end user changes from, say, $0.1 \%$ to say $0.01 \%$ cumulative, it can be readily translated into $z$, translated into a current density limit using (19), and then used in (11) for verification.

Fig. 7 shows a graphical representation of such a retargeting using the proposed model from a representative cell. For ease of exposition, we represent our model at a fixed slew, as shown in Fig. 2(a).

In Fig. 7, curve a) represents the reliability at the baseline condition. If the FF requirement of the design changes and drops to $10 \%$ of the original, the curve slides down to curve b) due to reduction in EM capability at tighter FF requirement. The drop is not drastic as this specific IP is rms-currentlimited, rather than being limited by the average current density. Similarly, if the use voltage has a $150-\mathrm{mV}$ overdrive over the characterized value, the reliability is represented by curve c), which shows degraded reliability due to increased 


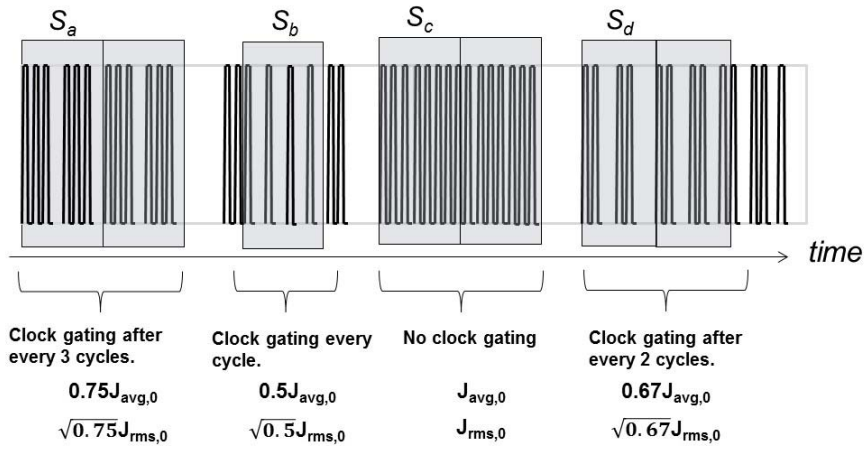

Fig. 9. Representative clock activity profile for a large duration. Different sampling windows show different activity rates (and corresponding $J_{\text {avg }}$ and $\left.J_{\mathrm{rms}}\right)$.

current flow. Similar behavior is shown in curve d) if the $\mathrm{JH}$ (rms current density specification) is tightened by $5{ }^{\circ} \mathrm{C}$. Finally, if the temperature requirement becomes $20{ }^{\circ} \mathrm{C}$ higher, design closure becomes more challenging with the reliability being now represented by the curve e) - almost $3 \times$ tightening.

The case study in Fig. 7 is handled very naturally in our approach. We reiterate that handling them in the traditional approach require a complete recharacterization of the $f_{\text {safe }}$ model at various conditions.

Next, to validate the retargeting methodology, we directly compare the curves of Fig. 7 to the curves of the traditional methodology (obtained by the actual characterization at the exact condition). As earlier, we present results from a single representative cell.

We show the percentage error for two conditions, curves c) and e) in Fig. 8. For curve e), where the temperature specification is altered, the required retargeting only affects the verification part (as the current density limits are scaled), which incurs little error. For curve c), the retargeting is due to $150-\mathrm{mV}$ overdrive, where we use a more approximate currentscaling model. The error here, although high, is acceptable, considering the fact that it is in a lower load regime (usually a low-current, EM-safe zone).

\section{B. Incorporating Nonuniform Clock Gating}

The case studies of Section V-A were helpful in outlining a general thought process on approaching the problem, when the eventual use scenario is different from the baseline one. We now consider the extension of those principles to the problem of clock gating, which was previously (Section IIIB3), analyzed under uniform assumption. In order to do so, a key input required is an activity profile of the design over several clock cycles, as shown in Fig. 9 [25]. We noted previously that the thermal time constant is a key determinant in addressing the non-uniform clock gating [26], [27], and any change in current profile (of a larger duration), should be handled individually, and cannot be combined as a timeweighted summation.

Hence, we follow a sliding window approach (with the duration as the thermal time constant), wherein the complete clock activity profile is scanned in a step-by-step manner. For every single time window scanned, we compute the effective activity rate, which can then be used to compute the cell-internal current densities through every resistor, arcs, and states of the cell. Eventually, for a resistor $R$, in the

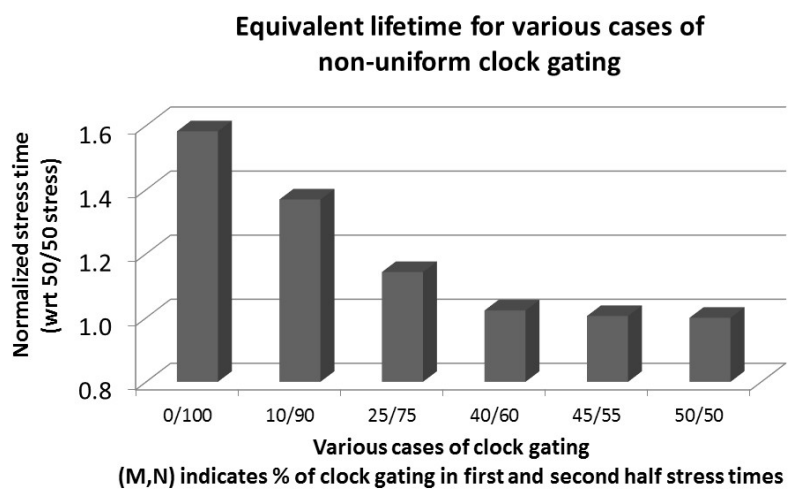

Fig. 10. Variation in reliability based on the extent of uniform clock gating in the first half and the second half of the stress time.

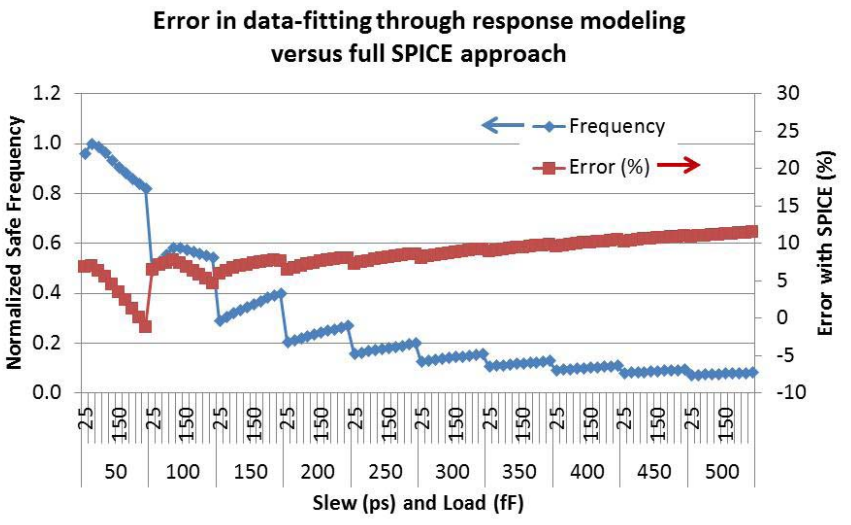

Fig. 11. Comparison of the response modeling approach (14) with full SPICE (red curve). $f_{\text {safe }}$ obtained through response modeling (blue curve).

$i$ th arc and $k$ th state, we can represent the current densities as: $\left\{\left(J_{\mathrm{avg}, R_{i k}, 0}, T_{0}\right), \ldots,\left(J_{\mathrm{avg}, R_{i k}, m}, T_{m}\right) ..\right\}$, where the index $m$ refers to the index of the sliding window. Clearly, every window can have a unique activity rate. For instance, in Fig. 9 , the sampling windows $S_{a}, S_{b}, S_{c}$, and $S_{d}$ correspond to a $75 \%$, $50 \%, 100 \%$, and $67 \%$ activity rate, respectively. This current stress can then be collapsed into a single equivalent stress, based on the concepts developed in the earlier discussions and using (19).

Indeed, for a variety of examples considering clock gating, we can notice a significant difference in the reliability. Fig. 10 shows the normalized reliability (of the clock-tree element) based on the extent and the nature of the clock gating.

For this experiment, we considered a single clock-tree element, which underwent different kinds of clock gating, though all amounting to a net $50 \%$ duration of gating in the chip lifetime. For example, the third column in Fig. 11 corresponds to a case where the clock remains $25 \%$ uniformly gated (meaning gated every one in four cycles) the first half of stress time, followed by $75 \%$ gated in the other half; and so on, for the other cases. After reliability computations based on (19), we plot the equivalent stress times for all cases, considering the $(50 \%, 50 \%)$ case as the baseline.

As we can see, for the same clock gating duration among all cases $(50 \%)$, the worst case reliability occurs for the case in which the full-throttle events are clustered togetherthereby meaning maximizing the average current, as well as $\mathrm{JH}$ together. On the other hand, if the clock gating is completely uniform, the $\mathrm{JH}$ is lowered, causing the least reliability loss. 


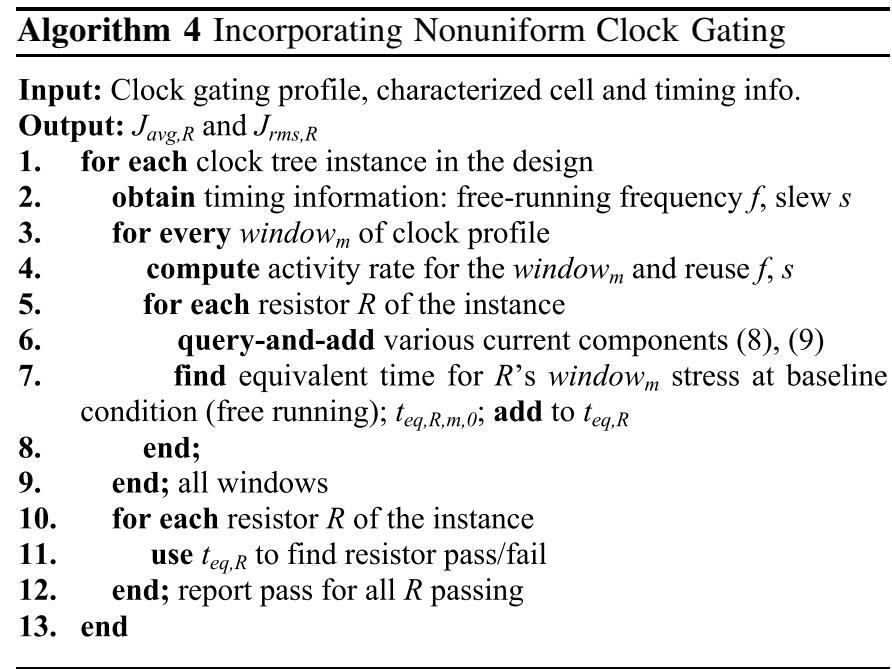

For the sake of completeness, we also note that for the same case, a free running clock corresponds to an equivalent lifetime of $\sim 3 \times$ as compared with the $(50 \%, 50 \%)$ case. Thus, we can capture the algorithm to incorporate the exact clock gating impact in Algorithm 4.

It must be mentioned that such a profiling data is hard to come by in real designs. Therefore, in absence of information, it is recommended to either assume no clock gating or assume clock gating in the nonuniform manner.

\section{Addressing L4: ACCElerated Data Generation USING CELL-RESPONSE MODELING}

Having looked at the various determinants of cell-EM reliability and ways to incorporate them in our model, we now look at expediting the characterization process. As discussed in Section III-C, for the traditional methodology, the safe frequency estimation requires 640 SPICE simulations per cell. Indeed, complete data generation for a production $28-\mathrm{nm}$ library, consisting of a few thousand cells, can run into days of effort. Such high runtimes for just a single baseline reliability condition make the process of EM characterization prohibitive under the traditional model. Although the efficiencies suggested earlier in this work can greatly reduce this overhead, it is still essential to use the baseline operating condition and characterize the current densities using (6) and (7): a process that can be very compute-intensive when carried out for all load/slew conditions. Hence, we must optimize the characterization process and in this work, we use response modeling approach

In the retargeting discussion of the last section, we noticed that the traditional methodology is inflexible, since it commingles the processes of current density computation and EM verification. For the same reason, it also does not lend itself for application of response modeling. The challenge here is twofold.

1) From the circuit point of view, operating parameters, such as load and slew nonuniformly, affect the individual rms and average resistor current densities in various arcs and states.

2) At the same time, the reliability specifications like lifetime and FF requirements nonuniformly influence the average and rms current-density thresholds.
Both of above eventually cause the average and rms-limited frequencies (discussed in the self-consistent estimation in Algorithm 2) to be asymmetrically impacted, thereby making the traditional frequency-level abstraction as nonscalable across load/slews (illustrated for example in Fig. 11) and reliability specifications (discussed earlier in Fig. 7).

On the other hand, a key feature of our approach is to keep characterization and verification disjoint, which presents an opportunity for model building during the characterization phase and accelerate the data generation process.

As noted in Section IV, average current flowing through the resistor is purely a function of the total charge transferred (lumped load), while the rms current density also has an inverse relationship with slew [3]. Based on these observations, we attempt to model the current density through any given resistor in the IP as a polynomial function of output loads/inputs slews

$J_{R_{j}}=a_{0}+b_{1} L+b_{2} L^{2}+c_{1} s+c_{2} s^{2}+d_{1} L s+d_{2} L^{2} s^{2}$.

Here, $a_{i}, b_{i}$, and $c_{i}$ are fitted coefficients, and $L$ and $s$ are the loads and slews, respectively. We identify seven critical points (in the $8 \times 8 \mathrm{load} / \mathrm{slew}$ matrix) that help to shape up the polynomial model: the four corners, $(1,1)$, $(1,8),(8,1)$, and $(8,8)$, and a few internal points $(2,4)$, $(4,4)$, and $(4,2)$, where the indices represent the index of the load and slew, respectively, in the table. The parameter fitting is then performed, based on (20), providing a model to predict the current densities at any arbitrary load/slew point. Note that the response modeling must be performed for every current density component (6)-(9) of the resistor $R$. The number of models corresponds to the total number of unique arcs and states of the cell. For example, for a singleinput clock-tree inverter, we would require a total of four simulations: two to cover the arcs (input rise to output fall, and vice versa), and two to cover the static states (input high and input low).

We now examine the validation of the response model for a representative IP cell in Fig. 11; the results from the entire library will be presented in an end-to-end manner in Section VII.

For various load/slew points on the $x$-axis, we first develop the characterization data based on the full SPICE simulations [using (6) and (7)]. Subsequently, the model from (20) is built using the simulation data from seven sampled points, and later evaluated at each of the $64 \mathrm{load} / \mathrm{slew}$ points. The normalized $f_{\text {safe }}$ is plotted on the left $y$-axis, and the error between model and SPICE is plotted on the right $y$-axis.

The nonmonotonic behavior of $f_{\text {safe }}$ with load/slew can be readily observed from this plot. Such nonmonotonicity arises from the fact that at different load/slew indices, the metal segments that limit the EM performance of the cell varies. For example, at a fixed load condition (say $100 \mathrm{fF}$ ), a lower input slew ( $\sim 25 \mathrm{ps}$ ) would mean a large rms current in the output signal resistors, while a smaller short-circuit current in the power-ground resistors. On the other hand, a higher input slew ( $\sim 200$ ps) means vice versa. Thus, for sharp input slews, the output signal resistors may often limit the cell reliability (due to rms constraint), while at the sluggish slews, the cell-internal power-ground resistors may be limiting (due to the average constraint). Such an interplay finally leads to a nonmonotonic 
TABLE I

RUNTIME COMPARISONS With PROPOSED AND TRADITIONAL Methods FOR A Single CELl

\begin{tabular}{lll}
\hline Methodology & $\begin{array}{l}\text { Simulations required } \\
\text { per cell }\end{array}$ & $\begin{array}{l}\text { Overall } \\
\text { compute }\end{array}$ \\
\hline $\begin{array}{l}\text { Traditional } \\
\text { (Fig. 2a) }\end{array}$ & $\begin{array}{l}\sim 640 \times n(n \text { : number of } \\
\text { design/reliability conditions })\end{array}$ & $\begin{array}{l}\sim 10 \text { mins. } \\
\text { per cell }\end{array}$ \\
\hline Proposed (eq. 20$)$ & $28(7$ load-slew $\times 4$ unique sims $)$ & $\sim 50 \mathrm{sec}$ \\
\hline
\end{tabular}

$f_{\text {safe }}$ behavior of the cell with load/slews.

Our methodology, however, only works at the current density level and, hence, remains unaffected by the reliability constraints that bring in the nonmonotonicity. Using the representation from (20) (for every resistor, per arc state), we can readily obtain the current densities at the chosen load/slew condition, and can subsequently use those to compute the safe frequency of the cell by using Algorithm 2, which additionally requires the reliability condition. Consequently, we can cover all the load/slew points to get the safe frequency plot, and as we can see, the response modeling approach works reasonably well in predicting the current densities and $f_{\text {safe, }}$, with an acceptable marginal error. Next, the runtime impact for a single cell is summarized in Table I.

As we see, the characterization runtime for our approach drops down significantly as compared with the traditional methodology, which takes $\sim 10 \mathrm{~min}$ to generate the safe frequency data for all load/slew points, whereas the proposed methodology (response modeling) is completed in about a minute. It must be mentioned here that the number of simulations required in the traditional methodology grows linearly with the number of design/reliability conditions required (as discussed in Section V). For example, every change in the voltage, stress temperature, or lifetime requires a new characterization. On the other hand, the proposed methodology comprehends the design/reliability conditions on the fly using the same database (Section V), hence further keeping down on the number of simulations required. Thus, the methodology in this section directly addresses the limitation L4 and significantly speeds up the characterization.

\section{Production Design Analysis}

We now examine the final application of the proposed methodology in an industrial scenario, discussing the setup and the workflow. A 28-nm high-performance block ( $2 \mathrm{~mm} \times 2 \mathrm{~mm}, \sim 600 \mathrm{k}$ instances, $>10 \mathrm{M}$ transistors), operating at $1-\mathrm{GHz}$ clock frequency is taken, which is part of a large industrial SoC. The entire flow is outlined in Fig. 12 for the proposed method.

The new method, in essence, is a three-step process: 1) IP characterization at a baseline reliability condition; 2) determining the reliability constraints for this design; and 3 ) integration into the timing/implementation tool. Note that the true retargeting flexibility of the proposed approach comes in form of 2), which is a runtime-level input to verification that is completely detached from 1 ). The flow of 3 ) uses a standard industrial design methodology.

\section{A. Library Characterization}

The entire library of a few thousand cells was characterized in two ways: 1) a full SPICE-based approach, where the

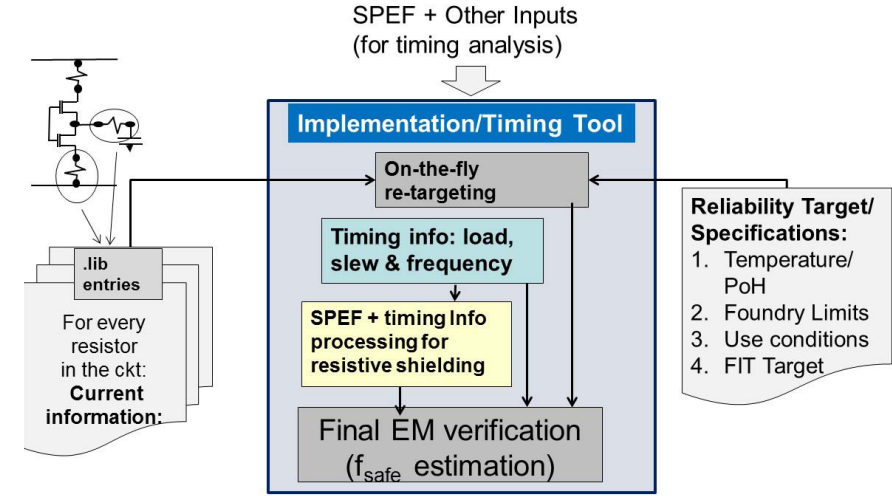

Fig. 12. Overall methodology and data-flow diagram for the proposed method.

traditional $f_{\text {safe }}$ table was generated at a baseline condition and 2) the methodology proposed in this paper. Parallelized and multithreaded SPICE simulations (using Cadence Spectre) were used. The runtime for 1) was $\sim 800 \mathrm{CPU}$ hours of raw simulation, excluding extraction, whereas the methodology in 2) completes in $\sim 80 \mathrm{CPU}$ hours. For 2 ), the production characterization framework for timing was used to arrive at the various arcs and logical states for switching and leakage current characterization.

\section{B. Final Reliability Verification}

The final application of the library-generated data was performed in the timing tool (Encounter Timing System), through a custom developed scriptware, which reads in both the characterization data types. The timing analysis of the design was performed at the baseline condition, to arrive at the slews and probabilistic switching rates through all the input pins. In the traditional approach, the scriptware steps through the timing information of every instance in the design and compares the queried $f_{\text {safe }}$ (from the traditional model) to the operating frequency. Note that since this approach suffers from the problems discussed earlier (specifically, L1 and L2), a final full SPICE simulation (with the $R C$ loading of the driver instance) is required after the initial results from the frequency comparisons. A total of $\sim 600 \mathrm{k}$ instances were analyzed in this way, and finally, the instances with the frequency ratio $>1$ (around 4500) were simulated further. The excitations for the SPICE simulations were a simple 1010 transition (at operating frequency), since all the instances were single-input clock-tree inverters, buffers, and gater cells (only eight unique cells). The final set of violations after the full SPICE simulations came down to 426 .

On the other hand, in the new approach, the scriptware additionally implements Algorithms 1-3, and based on the chip-level design and reliability conditions specifications (lifetime/temperature/voltage/FF), the equations are updated on the fly for the final frequency comparison of every instance.

Finally, we plot the population distribution of frequency ratios in Fig. 13. We consider five cases: curves a) and b) corresponding to analysis with the traditional and proposed methods at baseline reliability conditions, respectively, and curves c), d), and e) corresponding to the analysis at retargeted reliability conditions of a tighter $\mathrm{JH}$ limit, an overdrive case, and a high temperature requirement, respectively. 


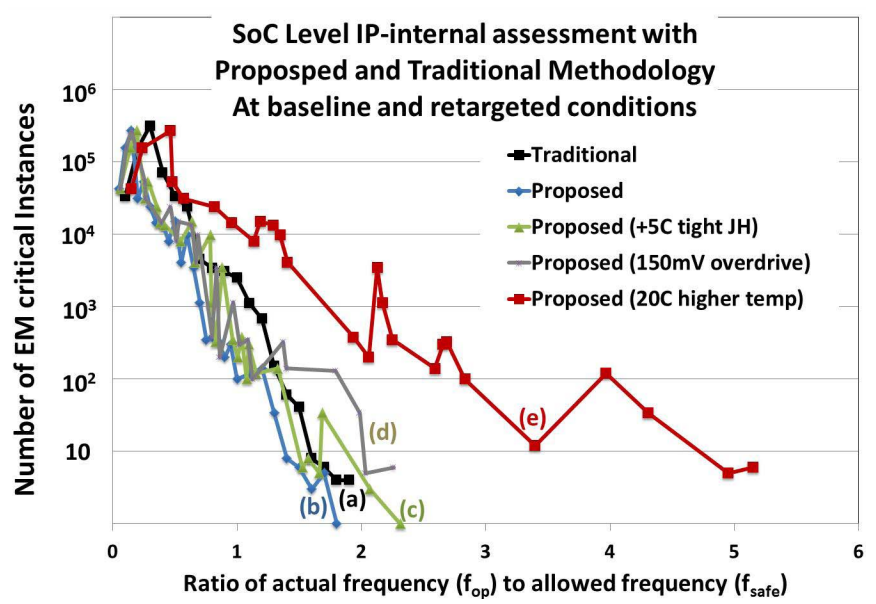

Fig. 13. Distribution plot for a $28-\mathrm{nm}$ block ( $>600 \mathrm{k}$ instances), highlighting the number of EM-critical instances and violations (with $f_{\text {op }} / f_{\text {safe }}$ ratio $>1$ ) for curves a) and b) baseline reliability analysis with the traditional and proposed methods and curves c), d), and e) retargeted reliability condition analysis with the proposed methodology.

\section{TABLE II}

Overall Comparison of Traditional Versus Proposed Methodology. Traditional Method WAS Run OnLY AT BASELINE CONDITION DUE TO RUNTIME ISSUES, Whereas the Proposed Method Could Run AT VARIOUS RELIABILITY CONDITIONS

\begin{tabular}{lll}
\hline Analysis Type & Reliability Condition & Violations \\
\hline Traditional (SPICE) & Baseline & 426 \\
\hline Proposed & Baseline & $442(421)$ \\
\cline { 2 - 3 } Methodology & Baseline +5C tighter JH & 1297 \\
\cline { 2 - 3 } & Baseline + 150mV overdrive & 1093 \\
\cline { 2 - 3 } & $\begin{array}{l}\text { Baseline + 20C higher } \\
\text { temperature requirement }\end{array}$ \\
\hline
\end{tabular}

For every method, we plot the ratio of $f_{\text {op }}$ to $f_{\text {safe, }}$, which signifies the EM criticality for that instance. Hence, an instance with $f_{\text {op }}$ greater than $f_{\text {safe }}$ (red region in the plot) is deemed as EM failure and must be acted upon for fixing (by either load reduction or replacement). The $y$-axis shows the distribution of number of instances in design with a particular $f_{\text {op }} / f_{\text {safe }}$ ratio. We document the total number of violations from various analyses in Table II.

As we can see from Fig. 13 and Table II, the proposed approach reports a total of 442 violations, 421 of which overlaps with the traditional methodology (+SPICE). The remaining false (21) and escaped (5) violations from the new approach were found to be relatively less critical, with frequency ratios in the range of 1.14-0.9. Thus, the new approach agrees well with SPICE.

Next, we demonstrate the final retargetability of the proposed approach is evident by the curves c), d), and e) in Fig. 13, analyzed at retargeted reliability conditions. Run curve c), corresponding to an additionally tight constraint of $5{ }^{\circ} \mathrm{C}$ lower $\mathrm{JH}$, results in almost three times increased violations, due to tighter rms limits. Run curve d), which is at overdrive conditions results in a similar violation profile. However, run curve e), which corresponds to a $20{ }^{\circ} \mathrm{C}$ higher stress temperature run results in a plethora of violations. Such a run is a close proxy to a direct application of IPs meant for handheld businesses to harsher environments!
Finally, based on the stage of the chip-design execution, design community has multiple ways to act upon this EM verification feedback. Although a detailed solution to developing EM fixes is beyond the scope of this paper, we provide some pointers in the rest of this paragraph. In many cases, the harshness of reliability criterion softens due to a lower lifetime requirement-for instance, in infotainment category chips [29]. Alternatively, an avoidance strategy can be followed upfront, wherein, based on the logic, high drive-strength cells are used to drive large fanout points. However, this requires careful consideration, since unwarranted improvement in drive-strength is associated with sharp output-slew reduction resulting in increased rms currents. On the other hand, a forceful lowering of drive-strength for instances with timing slack causes slew degradation resulting in increased short-circuit currents. A better approach may be through fanout load or activity reduction, which predictably reduces the current flow.

\section{CONCLUSION}

In summary, an accurate and retargetable methodology for IP-internal EM verification was presented in this paper. Generic switching rates for various pins of the IP are comprehended, including aspects of clock gating. Significantly high accuracy, with respect to SPICE, was achieved by incorporating the impact of arbitrary parasitic loading and an intelligent way of coming up with the effective pin capacitance of load cells. The methodology was shown to be highly flexible, in terms of allowing on-the-fly retargeting for the reliability. Finally, the complete data generation process at library level is expedited by application of cell-response modeling. Results on a 28-nm production setup were shared, to demonstrate significant relaxation in terms of violations, along with close correlation to SPICE. We shared various cases of runtime-level reliability retargeting by specifying varying reliability conditions for the production block verification. The methodology presented in this paper is most suitable in a third-party-IP context. The need is only underlined further with the increasing porting of designs from one business segment to a different one, which requires on-the-fly assessment of the reliability of all the components.

\section{REFERENCES}

[1] J. Lienig, "Electromigration and its impact on physical design in future technologies," in Proc. ACM Int. Symp. Phys. Design, 2013, pp. 33-40.

[2] X. Huang, T. Yu, V. Sukharev, and S. X.-D. Tan, "Physics-based electromigration assessment for power grid networks," in Proc. ACM/EDAC/IEEE Design Autom. Conf., Jun. 2014, pp. 1-6.

[3] P. Jain and A. Jain, "Accurate current estimation for interconnect reliability analysis," IEEE Trans. Very Large Scale Integr. (VLSI) Syst., vol. 20, no. 9, pp. 1634-1644, Sep. 2012.

[4] ITRS Interconnect Summary. [Online]. Available: http://www.itrs.net, accessed 2013.

[5] C. Yuan, D. Tipple, and J. Warner, "Optimizing standard cell design for quality," Proc. SPIE, vol. 9053, p. 90530O, Mar. 2014.

[6] JEDEC Reliability Standards, JC14. [Online]. Available: http://www. jedec.org

[7] AEC Q100 Specification, AEC-Q003 Rev-A. [Online]. Available: http://www.aecouncil.com/AECDocuments

[8] Encounter Design Implementation Tool User Manual. [Online]. Available: http://www.cadence.com, accessed 2014.

[9] Magma-Talus Implementation Tool User Manual. [Online]. Available: http://www.synopsys.com, accessed 2014.

[10] W. R. Hunter, "Self-consistent solutions for allowed interconnect current density-Part II. Application to design guidelines," IEEE Trans. Electron Devices, vol. 44, no. 2, pp. 310-316, Feb. 1997. 
[11] J. R. Black, "Electromigration failure modes in aluminum metallization for semiconductor devices," Proc. IEEE, vol. 57, no. 9, pp. 1587-1594, Sep. 1969

[12] K.-D. Lee, "Electromigration recovery and short lead effect under bipolar- and unipolar-pulse current," in Proc. IEEE Int. Rel. Phys. Symp., Apr. 2012, pp. 6B.3.1-6B.3.4.

[13] Altos Tool User-Manual. [Online]. Available: http://www.cadence.com, accessed 2012.

[14] S. S. Sapatnekar, Timing. New York, NY, USA: Springer, 2004.

[15] S. P. McCormick, "Modeling and simulation of VLSI interconnections with moments," Ph.D. dissertation, Dept. Elect. Eng. Comput. Sci., MIT, Cambridge, MA, USA, 1989.

[16] J. Qian, S. Pullela, and L. Pillage, "Modeling the "effective capacitance' for the RC interconnect of CMOS gates," IEEE Trans. Comput.-Aided Design Integr. Circuits Syst., vol. 13, no. 12, pp. 1526-1535, Dec. 1994

[17] C. C. Wang and D. Markovic, "Delay estimation and sizing of CMOS logic using logical effort with slope correction," IEEE Trans. Circuits Syst. II, Exp. Briefs, vol. 56, no. 8, pp. 634-638, Aug. 2009.

[18] J. F. Croix and D. F. Wong, "Blade and razor: Cell and interconnect delay analysis using current-based models," in Proc. ACM/IEEE Design Autom. Conf., Jun. 2003, pp. 386-389.

[19] S. Gupta and S. S. Sapatnekar, "Compact current source models for timing analysis under temperature and body bias variations," IEEE Trans. Very Large Scale Integr. (VLSI) Syst., vol. 20, no. 11, pp. 2104-2117, Nov. 2012.

[20] D. Sinha, S. Abbaspour, A. Bhanji, and J. M. Ritzinger, "Method of employing slew dependent pin capacitances to capture interconnect parasitics during timing abstraction of VLSI circuits," U.S. Patent 8103997 , Jan. 24, 2012

[21] B. Mullen, "CCS-timing: Composite current source delay modeling," in Proc. DAC, 2005.

[22] D. D. Ling, C. Visweswariah, P. Feldmann, and S. Abbaspour, "A moment-based effective characterization waveform for static timing analysis," in Proc. 46th ACM/IEEE Design Autom. Conf., Jul. 2009, pp. 19-24.

[23] HSPICE User Manual. [Online]. Available: http://www.synopsys.com

[24] S. Herbert and D. Marculescu, "Analysis of dynamic voltage/frequency scaling in chip-multiprocessors," in Proc. ACM/IEEE Int. Symp. Low Power Electron. Design, Aug. 2007, pp. 38-43.

[25] PTPX Power Estimation Tool. [Online]. Available: http://www. synopsys.com

[26] J. Choi, C.-Y. Cher, H. Franke, H. Hamann, A. Weger, and P. Bose, "Thermal-aware task scheduling at the system software level," in Proc. ACM Int. Symp. Low Power Electron. Design, 2007, pp. 213-218.

[27] Y. Zhan, S. V. Kumar, and S. S. Sapatnekar, "Thermally aware design," Found. Trends Electron. Design Autom., vol. 2, no. 3, pp. 255-370, Mar. 2008.

[28] A. Todri and M.-S. Malgorzata, "A study of reliability issues in clock distribution networks," in Proc. IEEE Int. Conf. Comput. Design, Oct. 2008, pp. 101-106.

[29] Automotive Processors Overview. [Online]. Available: http:// www.ti.com/lsds/ti/processors/dsp/automotive_processors/overview.page, accessed Aug. 2015.

[30] K. Banerjee and A. Mehrotra, "Coupled analysis of electromigration reliability and performance in ULSI signal nets," in Proc. IEEE/ACM Int. Conf. Comput. Aided Design, 2001, pp. 158-164.

[31] Y.-J. Park, P. Jain, and S. Krishnan, "New electromigration validation: Via node vector method," in Proc. IEEE Int. Rel. Phys. Symp., May 2010, pp. 698-704.

[32] S. M. Alam, G. C. Lip, C. V. Thompson, and D. E. Troxel, "Circuit level reliability analysis of $\mathrm{Cu}$ interconnects," in Proc. 5th Int. Symp. Quality Electron. Design, 2004, pp. 238-243.

[33] Z. Guan, M. Marek-Sadowska, S. Nassif, and B. Li, "Atomic flux divergence based current conversion scheme for signal line electromigration reliability assessment," in Proc. IEEE Int. Interconnect Technol. Conf., May 2014, pp. 245-248.
[34] K.-D. Lee, "Electromigration critical length effect and early failures in $\mathrm{Cu}$ /oxide and $\mathrm{Cu} / \mathrm{low} \mathrm{k}$ interconnects," Ph.D. dissertation, Dept. Mater. Sci. Eng., Univ. Texas Austin, Austin, TX, USA, 2003.

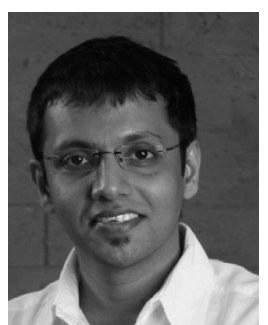

Palkesh Jain (M'04) received the bachelor's and master's degrees in electrical engineering from IIT Bombay, Mumbai, India, in 2004. He is currently pursuing the Ph.D. degree with the Universitat Politècnica de Catalunya, Barcelona, Spain.

He joined the ASIC Group, Texas Instruments India, Bangalore, India, where he defined and developed several gigahertz enabling reliability methodologies. Subsequently, he joined the Yield and Product Engineering team, Qualcomm Technologies Inc., Bangalore, in 2014, where he is involved in system level power and thermal management methodologies. He holds 15 U.S. patents (granted/pending).

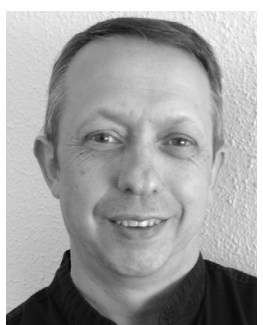

Jordi Cortadella (S'87-M'89-F'15) is currently a Professor with the Computer Science Department, Universitat Politècnica de Catalunya, Barcelona, Spain. His current research interests include formal methods and computer-aided design of VLSI systems with a special emphasis on asynchronous circuits, concurrent systems, and logic synthesis.

Prof. Cortadella is a member of Academia Europaea. He received best paper awards at the International Symposium on Advanced Research in Asynchronous Circuits and Systems in 2004, the Design Automation Conference in 2004, and the International Conference on Application of Concurrency to System Design in 2009. He has served on the technical committees of several international conferences in the field of design automation and concurrent systems, and is an Associate Editor of the IEEE TRANSACTIONS ON COMPUTER-AIDED DESIGN OF INTEGRATED CIRCUITS AND SYSTEMS.

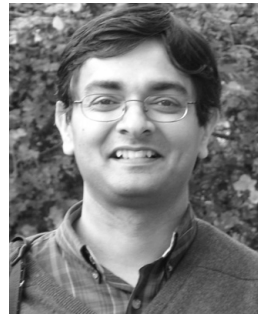

Sachin S. Sapatnekar (S'86-M'93-F'03) received the B.Tech. degree from IIT Bombay, Mumbai, India, the M.S. degree from Syracuse University, Syracuse, NY, USA, and the Ph.D. degree from the University of Illinois at Urbana-Champaign, Champaign, IL, USA.

He taught with Iowa State University, Ames, IA, USA, from 1992 to 1997. He has been with the University of Minnesota, Minneapolis, MN, USA, since 1997, where he holds the Distinguished McKnight University Professorship and the Robert and Marjorie Henle Chair.

Dr. Sapatnekar has received six conference best paper awards, the Best Poster Award, an ICCAD Ten-Year Retrospective Most Influential Paper Award, the SRC Technical Excellence Award, and the SIA University Researcher Award. 